\title{
TH6C-5
}

\section{Vector Mixer Characterization for Image Mixers}

\author{
Joel Dunsmore ${ }^{1}$, Sean Hubert ${ }^{1}$, Dylan Williams ${ }^{2}$ \\ ${ }^{1}$ Agilent Technologies, 1400 Fountaingrove Parkway, Santa Rosa, Ca, 95472 USA \\ ${ }^{2}$ NIST 818.01, 325 Broadway, Boulder, CO 80305, USA
}

\begin{abstract}
The vector mixer characterization method first introduced by Dunsmore can be used only for mixers where the Local Oscillator (LO) frequency lies between the RF input and output frequencies. Attempts to use the method for image mixers, where the LO is greater than both the input and output frequencies, yield erroneous results. An improved method is described which can properly characterize both types of mixers. A theoretical basis is introduced and experimental results are presented which justify the new method.
\end{abstract}

Index Terms - Frequency converters, group delay, image frequency, mixers, vector measurements.

\section{INTRODUCTION}

The accurate characterization of mixers and frequency converters for their amplitude, phase and group delay response has been an important though difficult challenge [1]. Recently, a new method was described by Dunsmore [2] that gives an accurate characterization of mixer response based on the application of reflection standards to the output of the mixer while monitoring the input return loss. However, the description and verification of this new method was applied only to RF mixers for which the Local Oscillator (LO) frequency lies between the mixer's input and output frequencies. We discovered that when the method is applied to image mixers, defined here as mixers for which the LO frequency lies above the mixer's input and output frequencies, the method gave erroneous results. The degree of the erroneous results depended upon the particular reflection standards used.

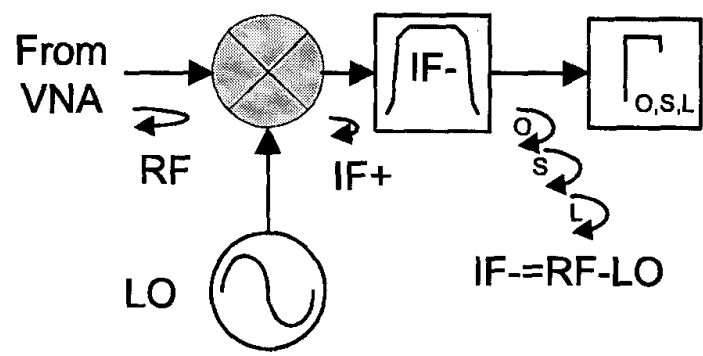

Fig. 1: Mixer characterization diagram. Three measurements are made: open (O), short (S), load response (L).
Figure 1 shows a block diagram of the measurement method, illustrating the application of the standards to a mixer. The method of [2] requires that some signal separation device such as a filter be applied at the output of the mixer to allow transmission of only one of the mixer products to the port where the reflection standards are applied. Figure 2 illustrates some of the possible mixer product combinations. The upper plots illustrate some combinations for an up-converter, where the input is marked $\omega_{I F}$. Note that two cases for the up-converter exist: the LO frequency can be below the output (called an RF mixer). This case is labeled as $\omega_{R F}$. Alternatively the LO can be above the output (called an image mixer); in this case the output is labeled $\omega_{I M}$, and is referred to as the image frequency.

The lower plots illustrate down-converters, where in one case the LO is at a lower frequency than the input (labeled $\omega_{R F}$ ), and in the other case the LO frequency is at higher frequency than the input (labeled $\omega_{M}$ ). As the arrows indicate in the figure, whenever the mixer creates an image frequency, and input is swept up in frequency, the direction of the output is reversed, resulting in a downward sweep. Reference [2] assumed that the filter from Fig. 1 would remove the image, in the case of up-

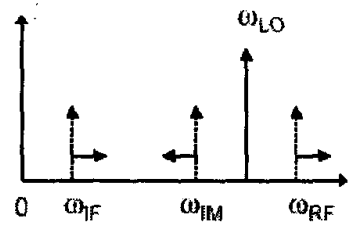

a) Changing IF, RF, of IA and fixed Lo.

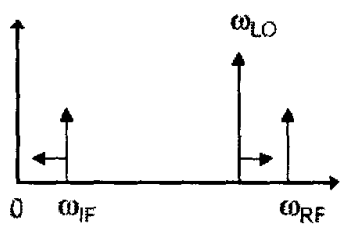

(c) Fixed RF and changing LO.

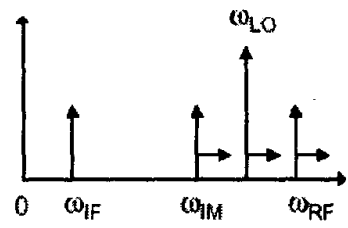

(b) Fixed IF and changing LO.

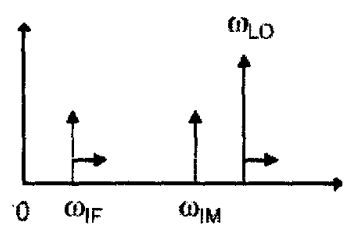

(d) Fixed INA and changing $L O$.
Fig. 2: Some of the possible mixer product combinations. (a) and (b) above show up-converters; (c) and (d) show down-converters 
converters, or that RF mixers would be used in the case of down-converters. However, many frequency converter applications exist that require that the image frequency be used.

\section{DISCOVERY OF THE ERRONEOUS RESULTS}

The experiments described in [2] were recently repeated for image mixers. These experiments yielded subtly erroneous results. The magnitude of the response appeared to be nearly correct, in that it substantially matched the amplitude response using power-meter based techniques. However the output match of the mixer, which is simultaneously determined in the method of [2], did not exactly match the measured $S_{22}$ of the mixer, when the $S_{22}$ was measured independently by using a vector network analyzer (VNA) with a load applied to the input. In particular, the phase was reversed from what was expected. While it is not clear that they should match in all cases, it is expected if the mixer can be treated as linear with respect to the small-signal input.

In the initial experiments, the reflection standards used were precision standards from a commercial calibration kit, referred to as mechanical standards. The open and short in this kit (Agilent 85033E) [3] are design to maintain an almost perfect 180 degree relationship over a wide range, and the load is nearly reflectionless. Enhancements in the method were made such that electronic calibration standards (ECAL) [3],[4] could be used. The ECAL module contains several reflection states that have a precisely known but arbitrary position on the Smith chart. When an ECAL module was used for the mixer characterization, the amplitude response was found to vary slightly from that obtained after calibration with the mechanical calibration kit, but the extracted $S_{22}$ response at the image port was found to

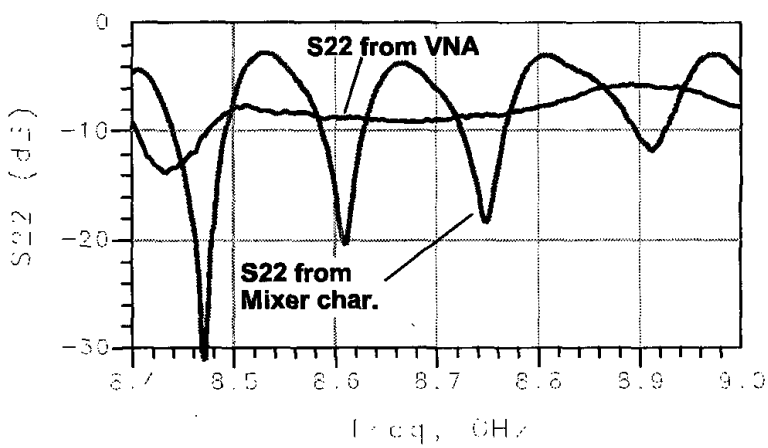

Figure 3: S22 from a direct VNA measurement (thin, black) and $\mathbf{S 2 2}$ extracted from a mixer characterization (thick, grey). vary wildly, when compared to the value measured directly on a VNA with a load applied at the RF port, as shown in Fig. 3. For some mixers, the extracted $S_{22}$ appeared greater than $0 \mathrm{~dB}$.

Clearly, it seemed there was some problem with ECAL method. Surprisingly, the same problem existed with the mechanical calibration kit. A final experiment was performed using a modified mechanical calibration kit, where an offset short was used, such that the relationship between the open and the short was no longer exactly 180 degrees. In this case, the $S_{22}$ also varied widely. The only condition under which a reasonable $S_{22}$ result could be obtained was one where the open and short had an ideally 180 degree phase relationship, and even then, the phase of $S_{22}$ was suspect. The fault appeared to lie in the method, not in the ECAL.

\section{SOLUTIONS FOR IMAGE-MIXER CHARACTERIZATION}

A clue to the cause of the difficulties in extracting the correct values for image mixers is found in the phase of the $S_{22}$ term. The $S_{22}$ phase of the mixer, as measured by a VNA, was found to have a negative slope as the applied output frequency was swept from low to high. This is entirely expected for return loss measurements. The phase of the $\mathrm{S}_{22}$ extracted from the characterization method (using mechanical standards) was also found to have a negative slope, even though the input was swept from low to high (thus the response at the output would sweep from high to low). Upon further thought, the phase should reverse in order to be consistent with the response, as measured using the VNA directly, so that the phase response is aligned over the entire frequency.

Thus, if the phase of the $S_{22}$ term of the mixer, as determined from reflections measured at the input port, should be reversed, its effect on the re-reflections of the standards applied would change depending upon the standards. For the case of the load, there is no change since the $S_{22}$ of the mixer does not affect the input reflection, because no signal is reflected from the load to interact with the $S_{22}$. In the case of the open and the short, the signal reflection from the standard is quite large, and interacts strongly with the $S_{22}$ of the mixer. The effective input impedance from a mixer terminated in a standard may be written as

$$
\Gamma_{\text {In }}=S_{11}^{I n}+\frac{C_{21} C_{12} \Gamma_{L}}{1-S_{22}^{O u t} \Gamma_{L}}
$$

where $S_{11}^{I n}$ is the input reflection of the mixer over the input frequencies; $C_{21}, C_{12}$ represent the complex conversion gain from input port to output port, and output port to input port over their respective frequencies; 
$S_{22}^{\text {out }}$ is the effective output impedance over the output frequencies, and $\Gamma_{L}$ is the load applied at the output. For the case where the reflection from the load can be ignored, and the open and short have unity reflection with exactly 180 degrees separation, the $S_{22}^{\text {Out }}$ term can be determined from

$$
S_{22}^{\text {out }}=\frac{\hat{\Gamma}_{o}+\hat{\Gamma}_{S}}{\left(\hat{\Gamma}_{o}-\hat{\Gamma}_{S}\right) \cdot e^{j \theta}}
$$

where $\hat{\Gamma}_{D}, \hat{\Gamma}_{S}$ are the measured responses of the open and short standards, after subtracting $S_{11}^{I n}$ (which is the load response). Here the open and short standards have values of $1 \cdot e^{j \theta}$ and $1 \cdot e^{j(\theta+\pi)}$ where $\theta$ is some value of phase offset for the open. From equation (2), we can see that if the conjugates of the standards are used (replace $\theta$ with $-\theta$ ), the amplitude of $S_{22}^{\text {Out }}$ is not affected. However, from equation (1), if the standards are not idealized as described above, the calculation of both amplitude and phase of $S_{22}^{\text {out }}$ becomes dependent on the proper phase relationship of each standard being determined.

From the fact that the frequency associated with $S_{22}^{\text {out }}$ is reversed in direction, we inferred that replacing the value of the standards with their conjugates for arbitrary standards (that is, standards that are not ideal, as described in equation (2)), might result in the proper determination of conversion gain and mixer output match. Measurements were made in accordance with the method outlined in Fig. 1, but with the value of each standard replaced with its conjugate before extracting the values of the mixer characteristics. Fig. 4 shows a comparison of four measurements done on the same mixer. The first set was performed on just the mixer, using $\Gamma_{L}$ in one case and $\Gamma_{L}^{*}$ in the other case. The experiment was repeated for the same mixer but with an airline attached (similar to the experiments performed in Fig. 4 of [2]). If the mixer conversion is extracted correctly, we expect to see the conversion gain amplitude essentially unaffected by adding the airline. Fig. 4 shows there is substantial difference between the two measurements when using $\Gamma_{L}$, but there is essentially no difference when using $\Gamma_{L}^{*}$.

Independent verification of the conversion gain can be obtained through scalar measurements of the input and output amplitudes. In these scalar measurements, the VNA receiver is calibrated to measure power by using a power meter to characterize the VNA source

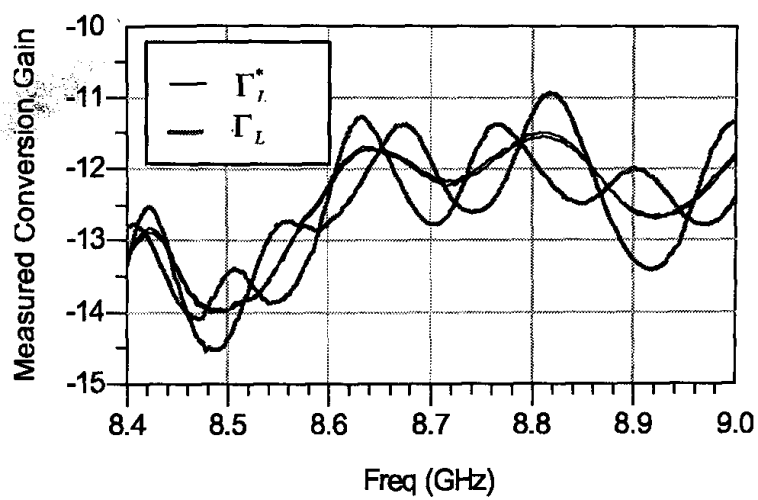

Fig. 4: Comparison of C21 of a mixer, and mixer with an added airline, for the case of normal standards ( 2 thick grey traces) and conjugate standards ( 2 thin black traces).

output power, which is in turn connected to the VNA measuring receiver so as to compare a reference source power applied to the receiver to the power level indicated by the receiver. As part of the calibration process, the complex impedance of the power meter, source and receiver are measured. From this we can correct for mismatch loss between these elements, yielding a highly accurate and traceable scalar (magnitude) measurement. This calibration technique is called Scalar Mixer Cal (SMC). If a scalar mixer measurement is done for both directions of the mixer (labeled $S C_{21}$ and $S C_{12}$ for scalar mixer conversion loss), the square-root of the product may be directly compared to the vector mixer measurement as shown in Fig. 5. The vector mixer technique characterizes the "round-trip" conversion loss of the mixer; that is, the square root of the product of $C_{21} * C_{12}$ (labeled $V C_{21}$ for vector conversion loss). The result for an image mixer using the conjugates of the reflection standards in calculations is also shown in Fig. 5. These two results compare very well, considering that entirely

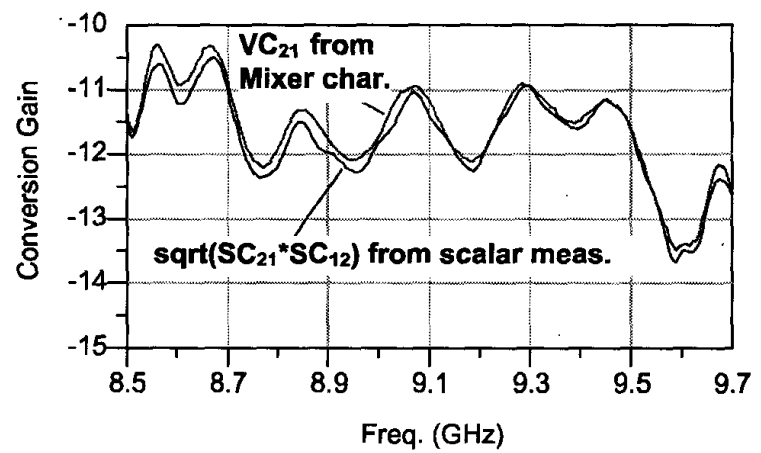

Fig. 5: Comparison of 2-way scalar measurements with the vector measurement using conjugate reflection standards. 
different techniques were used to arrive at these measurements. Further, measurements 'of $S_{22}^{\text {out }}$ using the conjugate of the reflections standards align almost perfectly with the same measurements made with a VNA, both in magnitude and phase, indicating a correct extraction of the mixer $S_{22}$.

\section{THEORETICAL CONSIDERATIONS}

Reference [5] develops a theoretical basis for the observed behavior, and applies traditional S-parameter definitions and techniques to the case of image mixers. In this case, the comparison was made with the Sparameters of a standard microwave junction, an RF mixer, and an image mixer. Though too detailed to reproduce for this paper, the result of these investigations determined a basis and some simple rules for dealing with signals applied to mixers, and interactions with other S-parameters networks. Consider the image mixer in Fig. 6, with applied waves as shown, which define an image mixer.

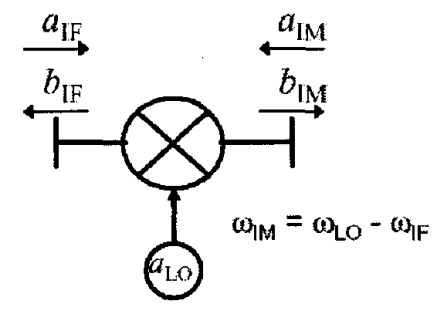

Fig. 6: An image mixer with applied waves.

Figure 7 shows some equivalent networks. Figure 7(b) shows that the effective input impedance of an RF mixer (where the LO frequency is low-side) terminated in a load can be computed in the standard way from S[IF] and $\Gamma_{L}$. For image mixers (where the LO is high-side), Fig. 7(c) shows that $\Gamma_{L}$, as it appears at the input of the mixer, must be conjugated. This is consistent with the measured results.

These examples demonstrate that to express the effect of $S_{22}^{M M}$ on the overall input measured reflection coefficient, it is necessary to take the conjugate of the output port reflection elements, just as was experimentally shown in Figs. 4 and 5. These abbreviated results are presented here only to illustrate that a mathematical basis exists for the experimentally derived procedure reported in this paper.

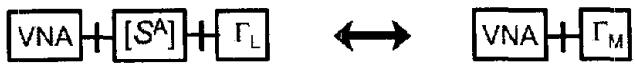

(a) Microwave junction

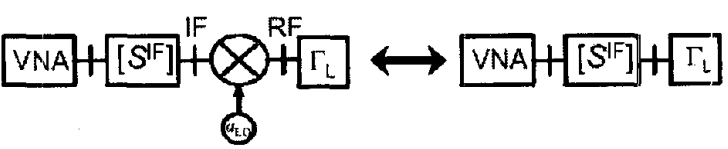

(b) RF Mixer "

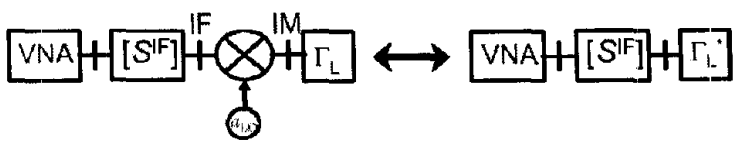

(c) Image Mixer

Fig. 7: Three examples which relate the measured response of parameters to the load applied to the output of a network.

\section{CONCLUSION}

With this paper the characterization of mixers using reflection standards has been extended to correctly account for the frequency and phase reversal effects of high-side mixers. This result extends the previous work of [2] where the effects of phase reversal were not considered, as the paper addressed only low-side mixers. The results described in this paper are somewhat unexpected, and may have consequences on behavioral models used for calculating mixer interactions with other microwave circuits.

\section{REFERENCES}

[1] C.J.Clark, A.A. Moultrop, M.S. Muha, C.P.Silva, "NNetwork Analyzer Measurement of Frequency-Translating Devices", Microwave Journal, Nov. 1996, pp 114-126.

[2] Dunsmore, J., "Novel Method for Vector Mixer Characterization and Mixer Test System Vector Error Correction", Proceedings of the IEEE MTT-S Symposium, June 2002

[3] We use trade names only to specify the experimental conditions. This does not imply an endorsement by the National Institute of Standards and Technology. Other products may work as well or better.

[4] User Guide for Agilent FCA application, PNA revision 4.05, Agilent user guide literature found at: http:/www.agilent.com/find/fca.

[5] D.F. Williams, F. Ndagijimana, K.A. Remley, J. Dunsmore, and S. Hubert, "Scattering-Parameter Models and Representations for Microwave Mixers", accepted for publication in IEEE Trans. Microwave Theory and Tech. 